\title{
Search for X-ray emission from subdwarf B stars with compact companion candidates
}

\author{
S. Mereghetti ${ }^{1}$, S. Campana ${ }^{2}$, P. Esposito ${ }^{3}$, N. La Palombara ${ }^{1}$, and A. Tiengo ${ }^{1,4}$ \\ 1 INAF, Istituto di Astrofisica Spaziale e Fisica Cosmica Milano, via E. Bassini 15, 20133 Milano, Italy \\ e-mail: sandro@iasf-milano.inaf.it \\ 2 INAF, Osservatorio Astronomico di Brera, via E. Bianchi 46, 23807 Merate (LC), Italy \\ 3 INAF, Osservatorio Astronomico di Cagliari, loc. Poggio dei Pini, strada 54, 09012 Capoterra (CA), Italy \\ ${ }^{4}$ IUSS, Istituto Universitario di Studi Superiori, viale Lungo Ticino Sforza 56, 27100 Pavia, Italy
}

Received 7 September 2011 / Accepted 24 October 2011

\section{ABSTRACT}

\begin{abstract}
Stellar evolutionary models predict that most early-type subdwarf stars in close binary systems have white dwarf companions. More massive companions, such as neutron stars or black holes, are also expected in some cases. Compact stars in these systems can be detected by means of X-rays powered by either accretion of the subdwarf stellar wind or surface thermal emission. Using the Swift satellite, we carried out a systematic search for X-ray emission from twelve subdwarf B stars that, based on optical studies, appear to have degenerate companions. None of our targets were detected, but the derived upper limits provide one of the few observational constraints on the stellar winds of early-type subdwarfs. Our results constrain the mass loss rates of some of these subdwarf B stars to values $\dot{M}_{\mathrm{W}}<10^{-13}-10^{-12} M_{\odot} \mathrm{yr}^{-1}$, if indeed they have neutron star companions.
\end{abstract}

Key words. subdwarfs - X-rays: binaries - stars: winds, outflows

\section{Introduction}

Subdwarf B (sdB) stars are believed to be low mass stars $\left(\sim 0.5 M_{\odot}\right)$ with a helium-burning core surrounded by a thin hydrogen layer (see Heber 2009, for a review of hot subdwarfs). A large fraction of known sdB stars are in close binary systems (Maxted et al. 2001; Morales-Rueda et al. 2003), supporting the idea that non-conservative mass transfer played a role in the loss of the massive hydrogen envelopes of these stars. Han et al. (2002) discussed different evolutionary channels leading to the formation of $\mathrm{sdB}$ binaries. These computations predict that the companions of sdB stars in short period systems ( $\sim 0.1-10$ days $)$ should fall into two main groups: late-type main-sequence stars or white dwarfs. More rarely, if the members of the original binary are massive enough, systems composed of a sdB and either a neutron star or black hole can also be formed.

These predictions about the nature of sdB binaries are difficult to test directly with optical observations. Most sdB are only single-lined spectroscopic binaries, because their bright optical/UV emission outshines that of their much fainter companions. However, the discovery and study of hot subdwarfs with compact companions is quite important because these systems might be among the progenitors of type Ia supernovae (Iben \& Tutukov 1994). Furthermore, determining the mass and evolutionary stage of the two components, as done e.g. for the binaries HD 49798 (Mereghetti et al. 2009, 2011) and KPD 1930+2752 (Geier et al. 2007), can shed light on the poorly known processes that take place during the common-envelope phases.

X-ray observations can help us to identify systems containing compact objects. X-rays can originate from either surface thermal emission of neutron stars or sufficiently hot white dwarfs, or can be produced if the compact object accretes mass from the sdB companion. Therefore, we carried out X-ray observations of a sample of $\mathrm{sdB}$ stars in close binary systems, for which evidence of a white dwarf, neutron star, or black hole companion had been found.

\section{Sample selection}

Several candidate subdwarfs with compact companions have been reported, based on radial and rotational velocity measurements of single-lined spectroscopic binaries (Geier et al. 2010). These authors showed that, with the reasonable assumption that the $\mathrm{sdB}$ star rotates synchronously with the orbital period, it is possible to derive the system inclination, hence to set a lower limit to the mass of its unseen companion. In many cases, the limits derived by Geier et al. (2010) exceed the masses of latetype main-sequence stars, implying that the companion is a white dwarf or, in a few cases, either a neutron star or a black hole.

Among the possible candidates, we considered those with the lowest interstellar absorption $\left(N_{\mathrm{H}}<5 \times 10^{20} \mathrm{~cm}^{-2}\right)$ and with shortest orbital period ( $0.1-0.8$ days). The latter condition yields a higher X-ray luminosity because in close binaries the compact object's orbit lies in a region where the mass donor's wind is denser and slower, thus giving rise to a higher accretion rate. Our targets are listed in Table 1, where we give some of their properties. The values for the estimated companion masses $M_{\mathrm{C}}$ (Col. 5) are those derived from the analysis of Geier et al. (2010). The $N_{\mathrm{H}}$ values reported in Table 1 correspond to the total Galactic column density ${ }^{1}$ in the source directions, hence they likely overestimate the actual interstellar absorption of our targets.

\footnotetext{
1 Derived from the on-line tool http://heasarc.nasa.gov/cgi-bin/Tools/w3nh/w3nh.pl, based on the works of Kalberla et al. (2005) and Dickey \& Lockman (1990).
} 
A\&A 536, A69 (2011)

Table 1. Main properties of the observed subdwarf binaries and results of Swift/XRT observations.

\begin{tabular}{lcccccccc}
\hline \hline Name & $\begin{array}{c}\text { Period } \\
(\text { days })\end{array}$ & $\begin{array}{c}\text { Distance } \\
(\mathrm{kpc})\end{array}$ & $\begin{array}{c}N_{\mathrm{H}} \\
\left(10^{20} \mathrm{~cm}^{-2}\right)\end{array}$ & $\begin{array}{c}M_{\mathrm{C}} \\
\left(M_{\odot}\right)\end{array}$ & Companion $^{a}$ & $\begin{array}{c}\text { Net exposure time } \\
(\mathrm{s})\end{array}$ & $\begin{array}{c}\text { Count rate }^{b} \\
\left(\mathrm{ct} \mathrm{s}^{-1}\right)\end{array}$ & $\begin{array}{c}L_{\mathrm{X}}{ }^{c} \\
\left(\mathrm{erg} \mathrm{s}^{-1}\right)\end{array}$ \\
\hline PG 1043+760 & 0.12 & 0.66 & 3.5 & $>0.1^{d}$ & WD & 5401 & $<3.8 \times 10^{-3}$ & $<7.3 \times 10^{30}$ \\
PG 1432+159 & 0.22 & 0.8 & 1.4 & $1.49-4.6$ & NS/BH & 3390 & $<3.8 \times 10^{-3}$ & $<9.8 \times 10^{30}$ \\
PG 2345+318 & 0.24 & 0.9 & 4.4 & $\ldots$ & WD & 3804 & $<4.0 \times 10^{-3}$ & $<1.5 \times 10^{31}$ \\
HE 0532-4503 & 0.27 & 2.8 & 3.3 & $2.08-3.94$ & NS/BH & 4729 & $<2.3 \times 10^{-3}$ & $<7.8 \times 10^{31}$ \\
CPD -64 481 & 0.28 & 0.21 & 4.3 & $0.38-1.04$ & WD & 4572 & $<3.8 \times 10^{-3}$ & $<7.9 \times 10^{29}$ \\
PG 1101+249 & 0.35 & 0.39 & 1.0 & $1.09-2.44$ & WD/NS/BH & 5263 & $<2.8 \times 10^{-3}$ & $<1.7 \times 10^{30}$ \\
PG 1232-136 & 0.36 & 0.57 & 3.4 & $>6$ & BH & 5589 & $<1.8 \times 10^{-3}$ & $<2.6 \times 10^{30}$ \\
GD 687 & 0.38 & 1.1 & 2.0 & $0.50-0.93$ & WD & 5149 & $<2.1 \times 10^{-3}$ & $<1.1 \times 10^{31}$ \\
HE 0929-0424 & 0.44 & 1.9 & 3.0 & $1.18-2.7$ & WD/NS/BH & 5566 & $<2.0 \times 10^{-3}$ & $<3.2 \times 10^{31}$ \\
PG 1743+477 & 0.52 & 1 & 2.5 & $>1.66$ & NS/BH & 3342 & $<4.3 \times 10^{-3}$ & $<1.8 \times 10^{31}$ \\
PG 0101+039 & 0.57 & 0.33 & 2.5 & $0.52-0.92$ & WD & 4851 & $<2.3 \times 10^{-3}$ & $<1.0 \times 10^{30}$ \\
TON S 183 & 0.83 & 0.54 & 1.2 & $0.63-1.33$ & WD & 4980 & $<2.1 \times 10^{-3}$ & $<2.5 \times 10^{30}$ \\
\hline
\end{tabular}

Notes. ${ }^{(a)} \mathrm{WD}=$ white dwarf, NS $=$ neutron star, $\mathrm{BH}=$ black hole. ${ }^{(b)} 3 \sigma$ upper limit in the $0.3-10 \mathrm{keV}$ energy range. ${ }^{(c)}$ In the $0.3-10 \mathrm{keV}$ range, corrected for the absorption. ${ }^{(d)}$ The presence of a white dwarf in this system with high inclination and short period is suggested by the non-detection of a variable reflection component in the light curve (Maxted et al. 2004). ${ }^{(e)}$ This sdB does not rotate synchronously, but the eclipse observed in this system indicates the presence of a white dwarf companion (Green et al. 2004).

\section{Observations and results}

The observations were carried out with the Swift/XRT telescope (Gehrels et al. 2004) between May and August 2011. All the data were obtained in photon counting mode. For most of our targets, the observations were split into a few short exposures, carried out over a time period spanning several days. The net exposure times, obtained by summing all the pointings of each target, are reported in Table 1.

No X-ray sources were detected in the Swift/XRT images at the positions of our targets. For each target, we calculated a $3 \sigma$ upper limit (following Gehrels 1986) based on the exposurecorrected count rate measured at the source position in the $0.3-10 \mathrm{keV}$ energy range, when also taking into account the instrumental effects on the point-spread function and the vignetting. The count rate upper limits were then converted into $\mathrm{X}$-ray fluxes, assuming a power-law spectrum with a photon index of two and the $N_{\mathrm{H}}$ values reported in Table 1 . The corresponding upper limits on the $0.3-10 \mathrm{keV}$ unabsorbed X-ray luminosities are typically in the range from $\sim 10^{30}$ to a few $\sim 10^{31} \mathrm{erg} \mathrm{s}^{-1}$. We used a power-law spectrum with the assumption that X-rays are produced by accretion. Thermal emission from neutron stars or hot white dwarfs would yield a much softer spectrum, peaking in the far UV/soft X-ray range, and having negligible flux above $\sim 0.3 \mathrm{keV}$ where Swift/XRT is most sensitive $^{2}$. The expected count rate in the case of thermal emission, would depend strongly on the interstellar absorption and on both the star's surface temperature and composition. Since the resulting upper limits do not provide particularly tight constraints, in view of the distances of our targets, we restrict the following discussion to the case of accretion-powered emission.

\section{Discussion}

The lack of X-ray detections for the sources in our sample does not allow us to confirm the presence of compact stars in these

\footnotetext{
2 For example, an extrapolation of the spectrum measured below $0.25 \mathrm{keV}$ for the hot white dwarf HZ43 (Beuermann et al. 2006) would give a $0.3-0.5 \mathrm{keV}$ count rate below $0.01 \mathrm{ct} \mathrm{s}^{-1}$ in the XRT. Scaling for the 3 to 40 times larger distances of our targets and considering even a modest interstellar absorption $\left(N_{\mathrm{H}}=10^{19} \mathrm{~cm}^{-2}\right.$, compared to $\sim 10^{18} \mathrm{~cm}^{-2}$ for HZ43) yields count rates between $10^{-6}$ and $3 \times$ $10^{-4} \mathrm{ct} \mathrm{s}^{-1}$.
}

systems. However, if we were to hypothesize that a collapsed object is indeed present in these binaries, our negative result could be used to constrain the poorly known properties of the sdB stellar winds. In all the systems we considered, accretion onto the compact object must indeed occur by means of stellar wind capture, since the $\mathrm{sdB}$ radius is much smaller than the Roche lobe.

Very little observational information is currently available about the mass-loss rate, $\dot{M}_{\mathrm{W}}$, from early type subdwarfs. Massloss rates of $\dot{M}_{\mathrm{W}} \sim 10^{-9}-10^{-8} M_{\odot} \mathrm{yr}^{-1}$ have been inferred from UV spectroscopy for a few O-type subdwarfs (Hamann et al. 1981; Hamann 2010). Lower values of $\dot{M}_{\mathrm{W}}$ are expected in sdB stars (Unglaub 2008), which are less luminous and have lower temperatures than sdO stars. Vink \& Cassisi (2002) derived an expression to compute the expected mass-loss rate for early-type stars as a function of their effective temperature, mass, luminosity, and metallicity. With the appropriate parameters for each sdB star of our sample (from Table 1 of Geier et al. 2010), and assuming solar metallicity, this relation yields values of $\dot{M}_{\mathrm{W}}$ in the range $\sim 10^{-12}-10^{-11} M_{\odot} \mathrm{yr}^{-1}$.

Our luminosity upper limits can be converted into limits on $\dot{M}_{\mathrm{W}}$ as follows. The expected mass accretion rate onto the compact object, $\dot{M}$, can be simply estimated, assuming Bondi-Hoyle accretion, from the relation

$\dot{M}_{\mathrm{W}} \pi R_{\mathrm{a}}^{2}=\dot{M} 4 \pi a^{2}$,

where $a$ is the orbital separation, and $R_{\mathrm{a}}=2 G M /\left(v_{\mathrm{o}}^{2}+v_{\mathrm{W}}^{2}\right)$ is the accretion radius, which depends on the mass $M$ of the accreting object, its velocity $v_{\mathrm{o}}$, and the velocity of the stellar wind $v_{\mathrm{W}}$. We computed the expected $\dot{M}$ of each source, using the parameters of Table 1 , a typical sdB mass of $0.45 M_{\odot}$, and conservatively assuming that $v_{\mathrm{W}}$ is equal to the $\mathrm{sdB}$ escape velocity. To compute $R_{\mathrm{a}}$ and convert luminosity into an accretion rate, we assumed for the white dwarf case a mass of $M=0.6 M_{\odot}$ and a radius $10^{9} \mathrm{~cm}$, while for the neutron star case we used $M=1.4 M_{\odot}$ and radius $10^{6} \mathrm{~cm}$. For PG 1232-136, in which the optical mass function suggests the presence of a black hole, we assumed a black hole of mass $6 M_{\odot}$ with an accretion efficiency of $10 \%$. The derived limits on $\dot{M}_{\mathrm{W}}$ are plotted in Fig. 1, where different symbols are used to indicate the assumed compact object.

The limits we obtained for the systems likely hosting white dwarfs (triangles in Fig. 1) are above $\dot{M}_{\mathrm{W}} \sim 5 \times 10^{-11} M_{\odot} \mathrm{yr}^{-1}$. 


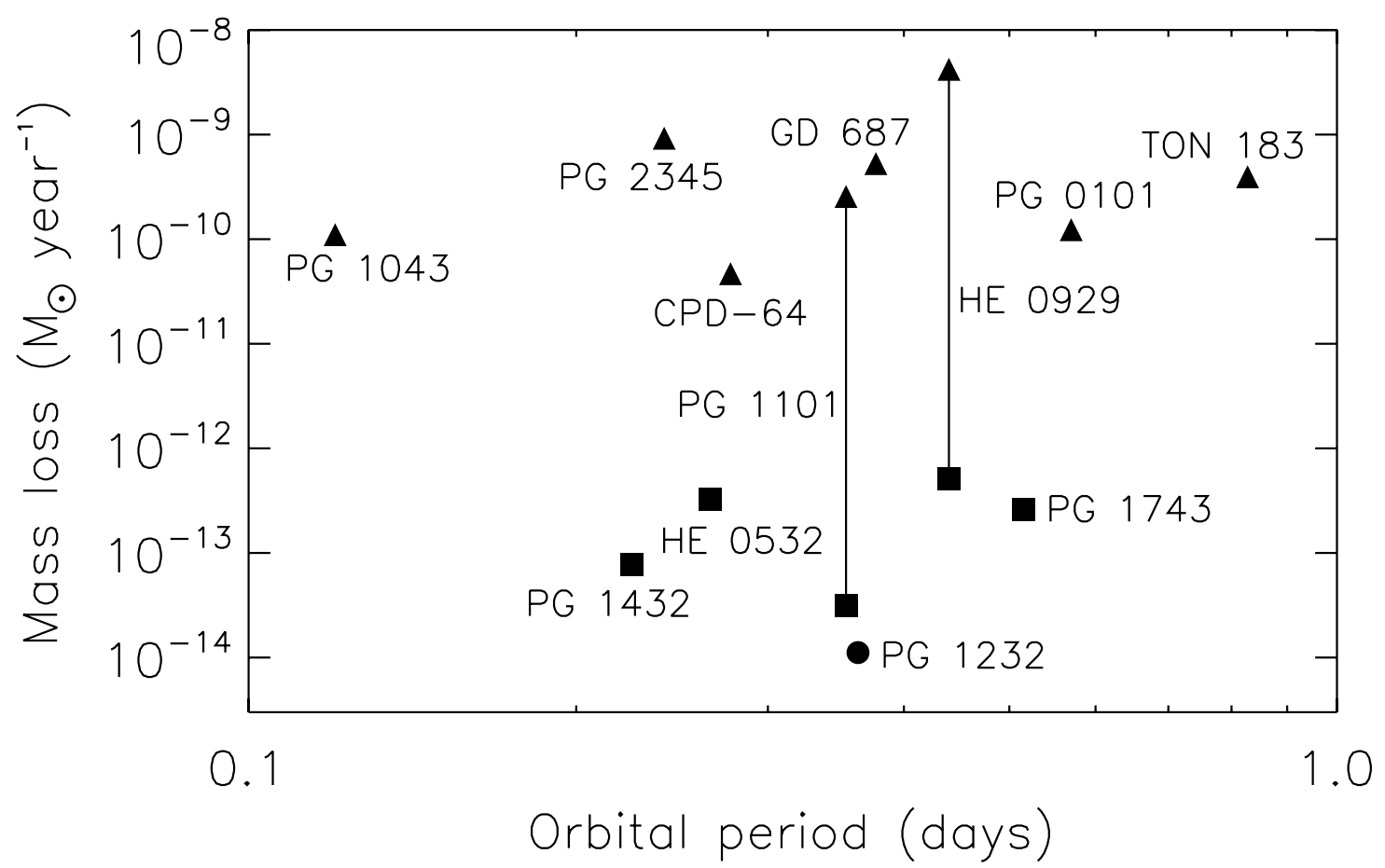

Fig. 1. Upper limits $(3 \sigma)$ on the sdB mass loss rates in the case of a white dwarf (triangles), a neutron star (squares), or a black hole (circle) companion.

Although these limits do not provide particularly tight constraints on the $\mathrm{sdB}$ wind properties, we note that they represent one of the few robust observational results in this field. Deeper X-ray observations of the closest candidates (e.g. PG 0101+039 and CPD -64 481) with more sensitive instruments will be able to detect accreting white dwarfs, if their sdB companions lose mass at a rate $\dot{M}_{\mathrm{W}} \sim 10^{-12}-10^{-11} M_{\odot} \mathrm{yr}^{-1}$, as predicted by theoretical wind models.

More interesting constraints can be inferred from the binaries likely containing neutron stars or black holes (PG $1432+159$, HE 0532-4503, PG 1232-136, and PG 1743+477). Of course, an obvious explanation of their non-detection is that some of the assumptions made to infer the presence of compact objects is wrong. We have not considered this possibility, but note that, as extensively discussed in Geier et al. (2010), it is rather unlikely. We also note that our simple estimate of the mass accretion rate in these systems leads to conservative upper limits on $\dot{M}_{\mathrm{W}}$. Most wind-accreting neutron stars in high mass X-ray binaries indeed have an X-ray luminosity higher than that predicted by Eq. (1). Therefore, we are quite confident that the lack of X-ray emission from these systems implies that the $\mathrm{sdB}$ stars have rather weak winds, with $\dot{M}_{\mathrm{W}}<3 \times 10^{-13} \quad M_{\odot} \mathrm{yr}^{-1}$. This is significantly below the predictions of the relation of Vink \& Cassisi (2002), if a solar metallicity is assumed. A metallicity $Z=0.3 Z_{\odot}$, or lower, is required for these $\mathrm{sdB}$ stars to be consistent with the derived upper limits.

\section{Conclusions}

Using the Swift/XRT telescope, we have carried out the first systematic search for accreting compact objects in binaries with B-type subdwarfs. None of the sdB binaries in our sample, which were selected based on optical studies that had suggested the presence of collapsed companions, was detected in the X-ray band, with typical luminosity upper limits of $\sim 10^{30}-10^{31} \mathrm{erg} \mathrm{s}^{-1}$. If the presence of neutron stars is confirmed in some of these systems, their lack of X-ray emission implies that the sdB mass-loss rates are lower than $\dot{M}_{\mathrm{W}} \sim$ $10^{-13} M_{\odot} \mathrm{yr}^{-1}$, below the predictions of theoretical models for radiatively driven stellar winds.

Acknowledgements. We thank the referee Martin Barstow for his useful suggestions. We acknowledge the use of public data from the Swift data archive. This work was partially supported with contributions from the agreements ASIINAF $I / 009 / 10 / 0$ and I/032/10/0. P.E. acknowledges financial support from the Autonomous Region of Sardinia through a research grant under the program PO Sardegna FSE 2007-2013, L.R. 7/2007 "Promoting scientific research and innovation technology in Sardinia”.

\section{References}

Beuermann, K., Burwitz, V., \& Rauch, T. 2006, A\&A, 458, 541 Dickey, J. M., \& Lockman, F. J. 1990, ARA\&A, 28, 215 Gehrels, N. 1986, ApJ, 303, 336

Gehrels, N., Chincarini, G., Giommi, P., et al. 2004, ApJ, 611, 1005

Geier, S., Nesslinger, S., Heber, U., et al. 2007, A\&A, 464, 299

Geier, S., Heber, U., Podsiadlowski, P., et al. 2010, A\&A, 519, A25

Green, E. M., For, B., Hyde, E. A., et al. 2004, Ap\&SS, 291, 267

Hamann, W. 2010, Ap\&SS, 329, 151

Hamann, W., Gruschinske, J., Kudritzki, R. P., \& Simon, K. P. 1981, A\&A, 104, 249

Han, Z., Podsiadlowski, P., Maxted, P. F. L., Marsh, T. R., \& Ivanova, N. 2002, MNRAS, 336, 449

Heber, U. 2009, ARA\&A, 47, 211

Iben, I. J., \& Tutukov, A. V. 1994, ApJ, 431, 264

Kalberla, P. M. W., Burton, W. B., Hartmann, D., et al. 2005, A\&A, 440, 775

Maxted, P. F. L., Heber, U., Marsh, T. R., \& North, R. C. 2001, MNRAS, 326, 1391

Maxted, P. F. L., Morales-Rueda, L., \& Marsh, T. R. 2004, Ap\&SS, 291, 307

Mereghetti, S., Tiengo, A., Esposito, P., et al. 2009, Science, 325, 1222

Mereghetti, S., La Palombara, N., Tiengo, A., et al. 2011, ApJ, 737, 51

Morales-Rueda, L., Maxted, P. F. L., Marsh, T. R., North, R. C., \& Heber, U. 2003, MNRAS, 338, 752

Unglaub, K. 2008, A\&A, 486, 923

Vink, J. S., \& Cassisi, S. 2002, A\&A, 392, 553 